\title{
铑催化的丙酮肟醚导向的苯酚的邻位烯基化反应
}

\author{
刘玲玲杨闪韩昳戴晨阳 \\ 史达清黄志斌* 赵应声* \\ (苏州大学材料与化学化工学部 苏州 215123)
}

摘要 以弱配位中心丙酮弜醚为导向基团, $\mathrm{Rh}$ 为催化剂, 实现了一种高区域选择性的苯酚类化合物邻位烯基化反应. 该 策略具有反应条件温和、底物适应性广、区域选择性好等优点. 为苯酚的选择性的邻位烯基化提供了一种新的有效方法. 关键词 苯酚; 丙酮肟醚; 烯基化

\section{Rhodium-Catalyzed ortho-Alkenylation of Phenols Directed by Acetone Oxime Ether}

\author{
Liu, Lingling Yang, Shan Han, Yi Dai, Chenyang \\ Shi, Daqing Huang, Zhibin* Zhao, Yingsheng* \\ (College of Chemistry, Chemical Engineering and Materials Science, Soochow University, Suzhou 215123)
}

\begin{abstract}
A practical rhodium-catalyzed highly regioselective ortho-alkenylation of phenolic compound under the assistance of weak coordination center acetoxime ether was developed. This strategy has advantages of simple and mild reaction conditions, wide scope of substrate and high regioselectivity. This protocol provids an efficient and new method for the regioselective ortho-alkenylation of phenols.
\end{abstract}

Keywords phenol; acetone oxime ether; alkenylation

近年来, 随着各种过渡金属催化的 $\mathrm{C}-\mathrm{H}$ 键官能团 化反应策略的报道 ${ }^{[1]}$, 苯酚的 $\mathrm{C}-\mathrm{H}$ 键官能团化反应研 究取得重大进展 ${ }^{[2]}$. C- $-\mathrm{H}$ 键的烯基化反应是在有机物分 子中引入碳-碳双键的有效方法, 许多有机化合物的 $\mathrm{C}-\mathrm{H}$ 键都可以进行选择性的烯基化 ${ }^{[3-16]}$. 邻烯基苯酚 是一系列天然产物合成的关键中间体 ${ }^{[17-19]}$, 因此发展高 效的实现苯酚的邻位选择性烯基化的策略具有重要的 意义. 苯酚传统的邻位烯基化的方法是通过 $o$-烯丙基苯 酚的 Claisen 重排来实现的 ${ }^{[20]}$. 但是, 这个方法的不足 在于很难控制反应的选择性, 可能会产生多位点取代产 物. 近年来, 通过导向基团辅助实现了苯酚的直接邻位 烯基化, 为苯酚的邻位烯基化提供了一类简单、高效的 路径 ${ }^{[21]} .2011$ 年, Gevorgyan 课题组 ${ }^{[22]}$ 报道了硅醚导向的
苯酚的邻烯基化反应，通过一锅两步法成功获得目标产 物. 同年, 刘否课题组 ${ }^{[23]}$ 报道了 $\mathrm{Rh}$ 催化的氨基甲酸酯 导向的苯酚的邻位烯基化反应. 2013 年, 刘桂霞和陆熙 炎等 ${ }^{[24]}$ 采用氧化性的导向基团成功实现了苯酚的邻位 烯基化反应. 虽然这些方法都高选择性地实现了苯酚的 邻位烯基化, 但是这些方法都存在一些不足, 例如有的 导向基团在反应过程中不易离去, 还需要后续转化才能 获得 $o$-烯基苯酚; 有的导向基团不易合成或者不稳定, 或价格昂贵. 这些都在一定程度上限制了这些方法的应 用. 因此, 发展一种简便高效的导向基团可以在反应过 程中自动脱除的一步直接获得邻烯基苯酚的策略显得 十分必要.

肜醚是一种重要的导向基团, 且肜梄结构广泛存在

\footnotetext{
* Corresponding authors. E-mail: zbhuang@suda.edu.cn; yszhao@suda.edu.cn Received April 13, 2020; revised May 22, 2020; published online May 29, 2020

Project supported by the National Natural Science Foundation of China (Nos. 21772139, 21572149), the Major Basic Research Project of the Natural Science Foundation of Jiangsu Higher Education Institutions (Nos. 15KJA150006, 17KJA150006), the Jiangsu Province Natural Science Found for Distinguished Young Scholars (No. BK2018g0041), the Project of Scientific and Technologic Infrasracture of Suzhou (No. SZS2018201708) and the Priority Academic Program Development of Jiangsu Higher Education Institutions Project.

国家自然科学基金(Nos. 21772139, 21572149)、江苏省高等学校自然科学基金重大基础研究(Nos. 15KJA150006, 17KJA150006)、江苏省杰出青年自然 科学基金(No. BK2018g0041)、苏州科技基础设施(No. SZS2018201708)和江苏高校优势学科建设工程资助项目.
} 
于天然产物中 ${ }^{[25-27]} .2015$ 年, 我们课题组 ${ }^{[28]}$ 就利用丙酮 肜醚为导向基团成功实现了苯甲醇的邻位烯基化反应. 丙酮肜醚在反应过程中易于脱除或转化为其它基团, 而 且合成丙酮肟醚所用的试剂都比较廉价, 有利于降低反 应的成本. 2019 年, 我们课题组再次利用肜醚为导向基 团成功实现了芳酮的邻位胺化反应 ${ }^{[29]}$ 及氧化环化反 应 $^{[30]}$, 成功构建了 $\mathrm{C}-\mathrm{N}$ 键. 作为这些工作的延续, 本 工作设计了弱配位中心丙酮肜醚为导向基团来实现苯 酚的选择性邻位直接烯基化. 该方法中导向基团丙酮肜 醚在反应过程中极易离去, 不需要切除操作便能一步直 接获得苯酚的邻位烯基化产物.

\section{1 结果与讨论}

\section{1 反应条件篮选}

$\mathrm{Rh}$ 是 $\mathrm{C}-\mathrm{H}$ 键烯基化反应常用的高效催化剂 $[31-34]$, 经过多次尝试, 我们发现 $\left[\mathrm{Cp}^{*} \mathrm{RhCl}_{2}\right]_{2}$ 是合适的催化剂. 以丙酮肜苯醚 $(1 \mathrm{a}, 0.2 \mathrm{mmol})$ 和丙烯酸乙酯 $(\mathbf{2} \mathrm{a}, 0.4$ $\mathrm{mmol}$ )为模板反应原料, 向其中加入 $5 \mathrm{~mol} \%$ 的 $\left[\mathrm{Cp} * \mathrm{RhCl}_{2}\right]_{2}, 1$ equiv. 的 $\mathrm{AgOAc}$ 以及 $1 \mathrm{~mL}$ 的三氟乙醇 $\left(\mathrm{CF}_{3} \mathrm{CH}_{2} \mathrm{OH}\right)$, 在 $60{ }^{\circ} \mathrm{C}$ 下反应 $14 \mathrm{~h}$, 最终以 $38 \%$ 的分离 产率获得产物 3a. 通过 ${ }^{1} \mathrm{H}$ NMR 等表征手段, 确定了 3a 的结构正是设想的目标产物一一苯酚的烯基化产物. 随 后, 对反应的溶剂和温度进行了篮选, 结果见表 1 . 探 究了多种非质子性溶剂和质子性溶剂对该反应的影响,

表 1 反应条件的篮选 ${ }^{a}$

Table 1 Screening of reaction conditions

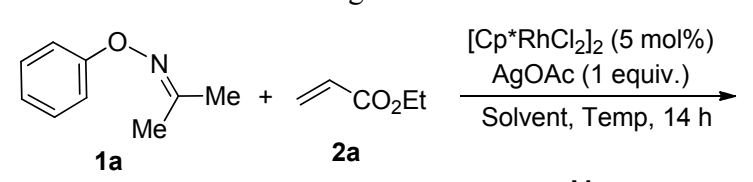

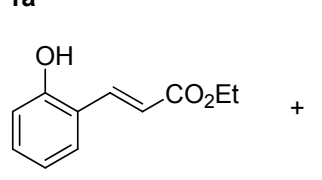

3a

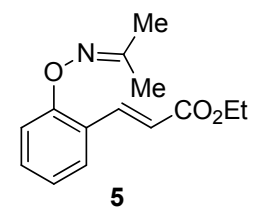

\begin{tabular}{cccc}
\hline Entry & Solvent & Temp. $/{ }^{\circ} \mathrm{C}$ & Yield $^{b} / \%(\mathbf{3 a} / \mathbf{5})$ \\
\hline 1 & $\mathrm{DCE}$ & 60 & 0 \\
2 & $\mathrm{THF}$ & 60 & 0 \\
3 & $1,4-\mathrm{Dioxane}$ & 60 & 0 \\
4 & $\mathrm{DMF}$ & 60 & 0 \\
5 & $\mathrm{CF}_{3} \mathrm{CH}_{2} \mathrm{OH}$ & 60 & $38 / 21$ \\
6 & $\mathrm{EtOH}$ & 60 & $8 / 0$ \\
7 & $\mathrm{HFIP}$ & 60 & $\mathrm{Trace}$ \\
8 & $\mathrm{MeOH}$ & 60 & $61 / 23$ \\
9 & $\mathrm{MeOH}$ & 25 & $0 / 87$ \\
10 & $\mathrm{MeOH}$ & 40 & $67 / 16$ \\
11 & $\mathrm{MeOH}$ & 80 & $86 / 0$ \\
12 & $\mathrm{MeOH}$ & 100 & $51 / 0$ \\
\hline
\end{tabular}

${ }^{a}$ Reaction conditions: 1a $(0.2 \mathrm{mmol}), 2 \mathrm{a}(0.4 \mathrm{mmol}),\left[\mathrm{Cp}^{*} \mathrm{RhCl}_{2}\right]_{2}(5 \mathrm{~mol} \%)$, AgOAc (1 equiv.), Solvent $(1 \mathrm{~mL}), 14 \mathrm{~h} ;{ }^{b}$ Isolated yields are given.
发现在非质子性溶剂中反应几乎不发生(表 1, Entries 1 4), 而在质子性溶剂中, 除在六氟异丙醇中只得到 极少量的产物外(表 1, Entry 7), 大多数醇中反应都可以 进行，其中甲醇的效果最好，产率高达 61\%(表 1, Entry 8). 在对温度进行考察的过程中, 意外地发现当反应在 $25{ }^{\circ} \mathrm{C}$ 下进行时, 获得了导向基团保留的产物 $\mathbf{5}$ (表 1 , Entry 9). 将反应温度逐渐升高, 体系中会出现两种产物 $3 \mathrm{a}$ 和 5 的混合物, 5 逐渐减少而 3a 逐渐增多 (表 1, Entries 8,10 ). 将温度升至 $80{ }^{\circ} \mathrm{C}$, 获得专一产物 $\mathbf{3 a}$ (表 1 , Entry 11). 温度继续升至 $100{ }^{\circ} \mathrm{C}$ 时, 产率降低至 $51 \%$ (表 1 , Entry 12). 通过以上条件篮选，得到了最优的反应条件： 催化剂 $\left[\mathrm{Cp}^{*} \mathrm{RhCl}_{2}\right]_{2}$ (5 mol\%), 添加剂 $\mathrm{AgOAc}$ (1 equiv.), 溶剂甲醇 $(1 \mathrm{~mL}), 80{ }^{\circ} \mathrm{C}$ 下反应 $14 \mathrm{~h}$.

\section{2 底物拓展}

在最佳反应条件下，对不同取代基团的丙酮肜苯醚 底物的适用性进行了考察，结果见表 2. 结果表明，无 论有吸电子还是供电子取代基，反应产率都会有所下 降. 当苯环间位被取代时，反应产率中等，并且反应发 生在位阻较小的邻位 $(\mathbf{3 b} \sim \mathbf{3 d})$. 当对位有丁基、卤素等

表 2 丙酮肜苯醚底物拓展 ${ }^{a}$

Table 2 Substrate expansion of acetoxime phenyl ether
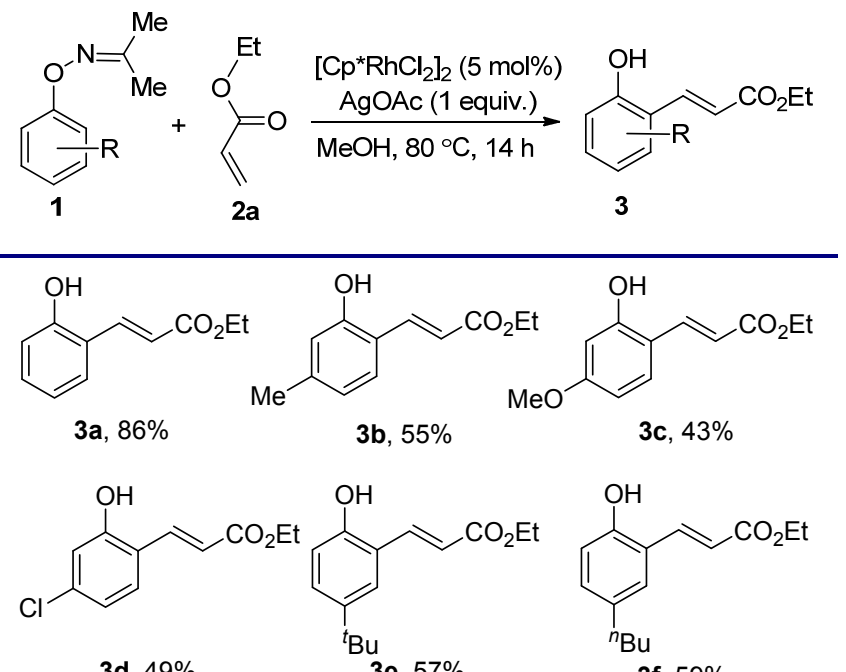

3d, $49 \%$

$3 e, 57 \%$

3f, $59 \%$<smiles>CCOC(=O)C=Cc1cc(Br)ccc1OCC=Cc1cc(Cl)ccc1O</smiles><smiles>CCOC(=O)/C=C/c1cccc(C(C)=O)c1O</smiles>

$3 \mathrm{j}, 51 \%$

$3 \mathbf{k}, 38 \%$

$31,46 \%$

${ }^{a}$ Reaction conditions: 1 (0.2 mmol), 2a $(0.4 \mathrm{mmol}),\left[\mathrm{Cp}^{*} \mathrm{RhCl}_{2}\right]_{2}(5 \mathrm{~mol} \%)$, $\mathrm{AgOAc}\left(1\right.$ equiv.), $\mathrm{MeOH}(1 \mathrm{~mL}), 14 \mathrm{~h} ;{ }^{b}$ Isolated yields are given. 
基团时 $(3 \mathrm{e} \sim 3 \mathbf{i})$, 反应获得中等至良好的产率. 而三氟 甲基取代基的底物(3j) 只有 51\%的收率，这可能是受电 子效应的影响. 此外, 当一个邻位被甲基占据时(31), 反 应产率降低至 46\%. 除了苯酚化合物还对联苯酚底物 (3k)进行了考察，可能受位阻效应影响，反应产率只有 $38 \%$. 值得一提的是, 所有的反应都得到了邻位烯基化 的单取代产物, 这可能是引入的双键及肟醚会和铑络合 物配位, 遏制进一步的邻位碳氢键活化, 导致只能得到 单邻位烯基化产物.

随后，在最优条件下对不同类型的烯烃底物进行了 考察, 结果见表 3 . 发现大多数吸电子烯烃都得到较好 的结果. 例如常见的丙烯酸酯类底物都有良好的适用 性, 以中等至良好的收率获得了目标产物 $(\mathbf{4 a} \sim \mathbf{4 e})$. 除 此之外, $N, N$-二甲基丙烯酰胺(4f) 和烯丙基砜底物(4g)也 适用于该反应, 并且获得了良好的收率. 另外, 多氟取 代和长链的丙烯酸酯类化合物 $(\mathbf{4 i} \sim \mathbf{4 j})$ 也获得了中等的 收率. 值得一提的是苯乙烯 $(4 \mathrm{~h})$ 作为反应物也监测到了 目标产物并且产率达到 48\%.

\section{表 3 烯烃底物拓展 ${ }^{a}$}

Table 3 Expansion of olefin substrate

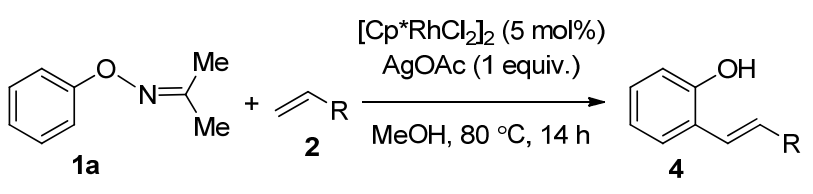

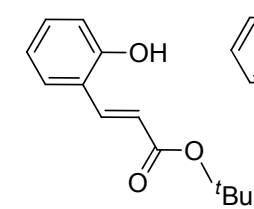

$4 a, 63 \%$<smiles>C=CCOC(=O)/C=C/c1ccccc1O</smiles>

4d, $64 \%$<smiles>CN(C)C(=O)/C=C/c1ccccc1O</smiles>

4f, $71 \%$

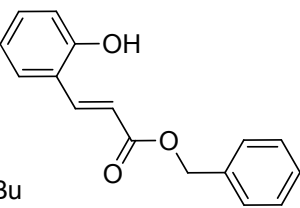

4b, $75 \%$

$4 e, 67 \%$<smiles>O=C(/C=C/c1ccccc1O)OCc1c(F)c(F)c(F)c(F)c1F</smiles>

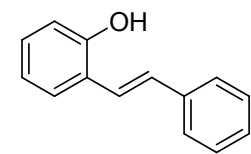

4h, $48 \%$

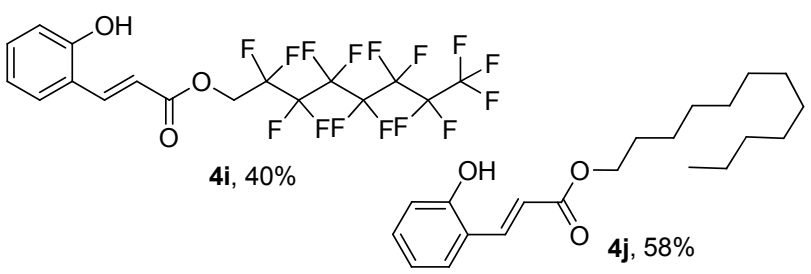

${ }^{a}$ Reaction conditions: 1a $(0.2 \mathrm{mmol}), 2(0.4 \mathrm{mmol}),\left[\mathrm{Cp}^{*} \mathrm{RhCl}_{2}\right]_{2}(5 \mathrm{~mol} \%)$,

$\mathrm{AgOAc}\left(1\right.$ equiv.), $\mathrm{MeOH}(1 \mathrm{~mL}), 14 \mathrm{~h} ;{ }^{b}$ isolated yields are given.

\section{3 克级反应}

为了考察反应的实用性，在标准条件下对模板反应 进行了克级规模的合成实验 (Scheme 1). 最终在 10 $\mathrm{mmol}$ 的反应中, 成功分离得到了 $1.21 \mathrm{~g}$ 产物 $\mathbf{3 a}$, 产率 为 $63 \%$. 虽然产率比之前的小剂量反应有所降低，但较 为良好的收率证明该反应具有一定的应用价值.

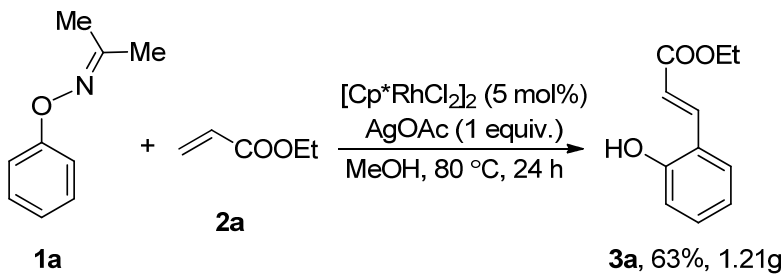

图 1 克级反应

Scheme 1 Gram reaction

\section{4 控制实验}

为深入探究反应的机制, 进行了控制实验 (Scheme 1). 结果表明, 当反应体系中不加 $\left[\mathrm{Cp}^{*} \mathrm{RhCl}_{2}\right]_{2}$ 时, 薄层 色谱 (TLC) 监测发现没有目标产物生成。这说明 $\left[\mathrm{Cp}^{*} \mathrm{RhCl}_{2}\right]_{2}$ 不仅是有效的催化剂而且对反应是必需的, 进而我们猜想反应中可能存在 $\mathrm{C}-\mathrm{H}$ 键活化过程. 接着 移除 $\mathrm{AgOAc}$, 我们发现反应同样不能进行, 由此可知 $\mathrm{AgOAc}$ 也是反应必需的. 为了验证反应过程是否存在 $\mathrm{C}-\mathrm{H}$ 键活化, 我们进行了动力学研究. 我们用全氛底 物和普通底物在标准条件下进行分子间的竞争反应，核 磁分析结果显示动力学同位素效应(KIE)值为 2.6, 因此 我们认为反应过程中发生了 $\mathrm{C}-\mathrm{H}$ 活化并且它的断裂可 能是决速步骤.
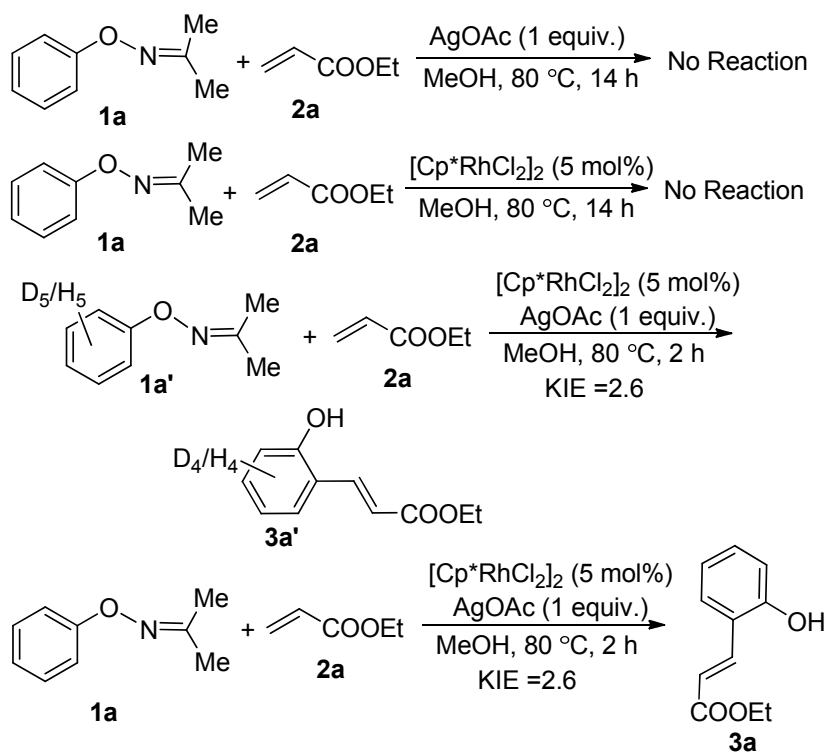

图式 1 控制实验

Scheme 1 Control experiment 
基于上述的实验事实和之前文献的报道 ${ }^{[35-36]}$, 我们 提出如下的催化循环过程(Scheme 2): 催化剂通过与乙 酸银进行阴离子交换生成活性催化剂后, 与底物结合, 生成中间络合物 $\mathbf{I}$, 然后烯烃迁移插入, 生成含有铑(III) 的七元环状络合物 II. 之后, 经历 $\beta-\mathrm{H}$ 消除, 生成了络 合物 III. 在醋酸作用下生成产物 3 .

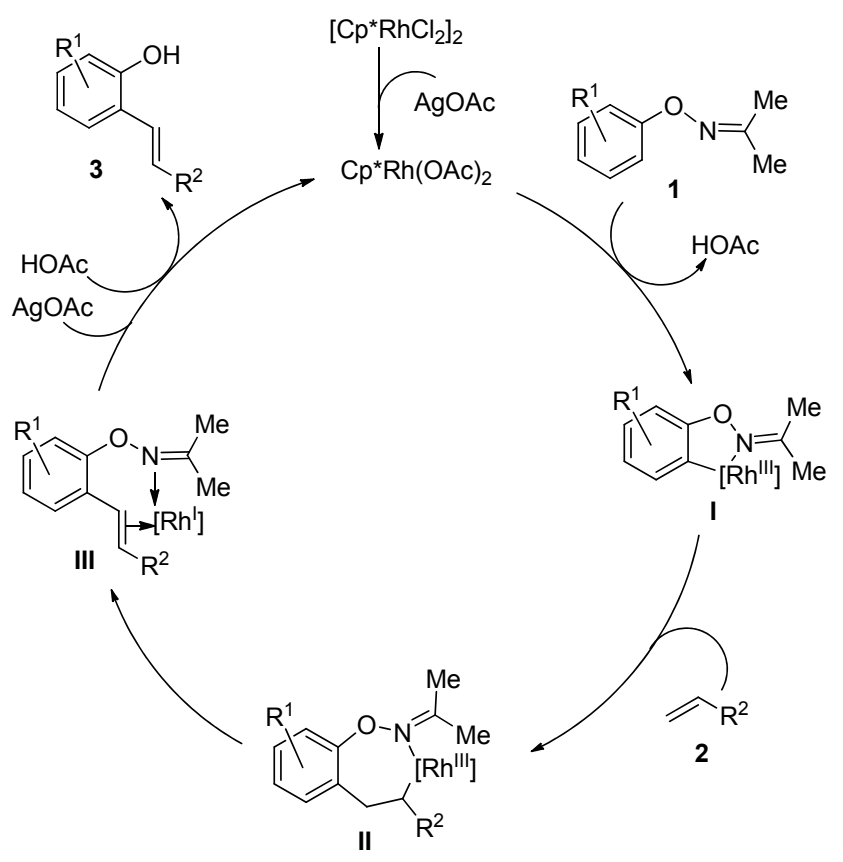

图 2 可能的机理

Scheme 2 Possible mechanism

\section{2 结论}

以丙酮肟醚为导向基团, 铑为催化剂, 实现了高效 的、高区域选择性的苯酚的邻位烯基化策略. 该方法具 有反应条件简单温和、底物适应性广、区域选择性好等 优点. 反应可以很好地放大到克级, 具有较好的应用价 值.

\section{3 实验部分}

\section{1 仪器与试剂}

${ }^{1} \mathrm{H} N M R 、{ }^{13} \mathrm{C} N M R 、{ }^{19} \mathrm{~F} N M R$ 的测试仪器为 Bruker $\mathrm{AV} 400 \mathrm{Mhz}$ 核磁共振仪, 采用 $\mathrm{CDCl}_{3}$ 和 $\mathrm{DMSO}-d_{6}$ 为気 代试剂, TMS 为内标; 高分辨质谱仪器为 Bruker MicrOTOF-Q II.

\section{2 化合物 1 的合成方法}

参考文献[37]方法, 向 $100 \mathrm{~mL}$ 的圆底烧瓶中加入 $10 \mathrm{mmol}$ 的 $N$-着基邻苯二甲酰亚胺和 $20 \mathrm{mmol}$ 的芳基喼

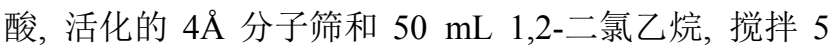
$\min$ 后向其中加入 $11 \mathrm{mmol}$ 氯化亚铜和 $0.9 \mathrm{~mL}$ 吡啶, 常 温下敞口搅拌 $48 \mathrm{~h}$, 薄层色谱(TLC)监测反应. 反应完
全后，绿色反应液通过硅藻土过滤，减压蒸馏除去溶剂 得粗产物, 粗产物用硅胶柱层析提纯得白色纯产物, 淋 洗剂为石油醚和乙酸乙酯的混合溶剂 $[V$ (石油醚) $: V($ 乙 酸乙酯 $)=10 ： 1]$. 将第一步制得的白色固体产物溶于 $30 \mathrm{~mL}$ 的二氯甲烷中, 加入 $40 \mathrm{mmol}$ 的水合肼，室温下 搅拌 $6 \mathrm{~h}$. 过滤反应液, 滤液用无水硫酸钠干燥, 滤出干 燥剂, 低温下旋干溶剂, 获得淡黄色液体产物. 向第二 步制得的淡黄色液体中加入溶剂量的丙酮，常温摚拌 6 h. 旋干溶剂得粗产物, 粗产物用硅胶柱层析提纯得纯 产物 $\mathbf{1}$, 淋洗剂为石油醚和乙酸乙酯的混合溶剂 $[V$ (石油 醚)： $V$ (乙酸乙酯 $)=20 ： 1$ 1]. 1a $\sim 11$ 均为已知化合物，表 征结果与文献[37]一致.

\section{3 化合物 3 和 4 的合成方法}

向 $15 \mathrm{~mL}$ 配有搅拌子的玻璃反应管中加入 $\left[\mathrm{Cp}^{*} \mathrm{RhCl}_{2}\right]_{2}$ (6.2 mg, $\left.5 \mathrm{~mol} \%\right), A g O A c(0.2 \mathrm{mmol}, 1$ equiv.), 丙酮肜醚 $(0.2 \mathrm{mmol}, 1$ equiv.), 烯烃 $(0.4 \mathrm{mmol}, 2$ equiv.)和 $1 \mathrm{~mL}$ 甲醇, 将反应管密封, 在 $80{ }^{\circ} \mathrm{C}$ 的油浴锅 中反应 $14 \mathrm{~h}$. 反应完全后, 减压旋干溶剂得粗产物, 粗 产物用硅胶柱层析提纯得纯产物 $\mathbf{3}$ 或 $\mathbf{4}$, 淋洗剂为石油 醚和乙酸乙酯的混合溶剂 $[V$ (石油醚) $: V$ (乙酸乙酯 $)=$ $8: 1]$.

(E)-3-(2-羟苯基)丙烯酸乙酯(3a): $33.0 \mathrm{mg}$, 白色固 体, 产率 86\%. m.p. $63 \sim 65{ }^{\circ} \mathrm{C}$ (文献值 ${ }^{[38]}$ : $64 \sim 65{ }^{\circ} \mathrm{C}$ ); ${ }^{1} \mathrm{H}$ NMR $\left(400 \mathrm{MHz}, \mathrm{CDCl}_{3}\right) \delta: 8.06 \sim 7.98(\mathrm{~m}, 1 \mathrm{H})$, $7.49 \sim 7.44(\mathrm{~m}, 1 \mathrm{H}), 7.25 \sim 7.21(\mathrm{~m}, 1 \mathrm{H}), 6.95 \sim 6.89(\mathrm{~m}$, $1 \mathrm{H}), 6.85(\mathrm{~d}, J=8.1 \mathrm{~Hz}, 1 \mathrm{H}), 6.66 \sim 6.59(\mathrm{~m}, 1 \mathrm{H}), 6.51(\mathrm{~s}$, $1 \mathrm{H}), 4.29$ (q, $J=7.1 \mathrm{~Hz}, 2 \mathrm{H}), 1.35(\mathrm{t}, J=7.1 \mathrm{~Hz}, 3 \mathrm{H}) ;{ }^{13} \mathrm{C}$ NMR $\left(101 \mathrm{MHz}, \mathrm{CDCl}_{3}\right) \delta: 168.8,155.7,141.0,131.4$, 129.1, 121.6, 120.4, 118.0, 116.4, 60.7, 14.2; HRMS (ESI) calcd for $\mathrm{C}_{11} \mathrm{H}_{12} \mathrm{NaO}_{3}[\mathrm{M}+\mathrm{Na}]^{+}$215.0684, found 215.0670.

(E)-3-(2-羟基-4-甲基苯基)丙烯酸乙酯(3b)：22.7 $\mathrm{mg}$, 白色固体, 产率 55\%. m.p. $87 \sim 88{ }^{\circ} \mathrm{C}$ (文献值 ${ }^{[39]}$ : $\left.88 \sim 90{ }^{\circ} \mathrm{C}\right) ;{ }^{1} \mathrm{H}$ NMR (400 MHz, $\left.\mathrm{CDCl}_{3}\right) \delta: 8.01(\mathrm{~d}, J=$ $16.1 \mathrm{~Hz}, 1 \mathrm{H}), 7.35$ (d, J=7.9 Hz, 1H), 6.84 6.70 (m, 2H), $6.68(\mathrm{~s}, 1 \mathrm{H}), 6.60(\mathrm{~d}, J=16.1 \mathrm{~Hz}, 1 \mathrm{H}), 4.29$ (q, $J=$ $7.1 \mathrm{~Hz}, 2 \mathrm{H}), 2.30(\mathrm{~s}, 3 \mathrm{H}), 1.35(\mathrm{t}, J=7.1 \mathrm{~Hz}, 3 \mathrm{H}) ;{ }^{13} \mathrm{C}$ NMR (101 MHz, $\left.\mathrm{CDCl}_{3}\right) \delta: 168.9,155.6,142.3,141.0$, 129.0, 121.5, 118.9, 117.0, 116.9, 60.6, 21.3, 14.2; HRMS (ESI) calcd for $\mathrm{C}_{12} \mathrm{H}_{14} \mathrm{NaO}_{3}[\mathrm{M}+\mathrm{Na}]^{+}$229.0841, found 229.0831.

(E)-3-(2-着基-4-甲氧基苯基)丙烯酸乙酯(3c)：19.1 $\mathrm{mg}$, 白色固体, 产率 43\%. m.p. 120 $121{ }^{\circ} \mathrm{C}$ (文献值 ${ }^{[38]}$ : $\left.118 \sim 120{ }^{\circ} \mathrm{C}\right) ;{ }^{1} \mathrm{H}$ NMR $\left(400 \mathrm{MHz}, \mathrm{CDCl}_{3}\right) \delta: 8.21(\mathrm{~d}, J=$ $16.4 \mathrm{~Hz}, 1 \mathrm{H}), 7.56(\mathrm{~s}, 1 \mathrm{H}), 7.18 \sim 7.11(\mathrm{~m}, 1 \mathrm{H}), 7.02(\mathrm{~d}$, 
$J=16.4 \mathrm{~Hz}, 1 \mathrm{H}), 6.55 \sim 6.50(\mathrm{~m}, 1 \mathrm{H}), 6.45(\mathrm{~d}, J=8.2 \mathrm{~Hz}$, $1 \mathrm{H}), 4.30$ (q, $J=7.1 \mathrm{~Hz}, 2 \mathrm{H}), 3.86$ (s, 3H), 1.36 (t, $J=7.1$ $\mathrm{Hz}, 3 \mathrm{H}) ;{ }^{13} \mathrm{C}$ NMR $\left(101 \mathrm{MHz}, \mathrm{CDCl}_{3}\right) \delta: 169.7,160.1$, 157.3, 136.5, 131.2, 119.9, 111.0, 108.8, 102.6, 60.5, 55.6, 14.3; HRMS (ESI) calcd for $\mathrm{C}_{12} \mathrm{H}_{14} \mathrm{NaO}_{4}[\mathrm{M}+\mathrm{Na}]^{+}$ 245.0790, found 245.0791 .

(E)-3-(4-氯-2-羟基苯基)丙烯酸乙酯 (3d): $22.2 \mathrm{mg}$, 白色固体, 产率 $49 \%$. m.p. $108 \sim 111{ }^{\circ} \mathrm{C}$ (文献值 ${ }^{[40]}$ : $\left.109 \sim 110{ }^{\circ} \mathrm{C}\right) ;{ }^{1} \mathrm{H}$ NMR $\left(400 \mathrm{MHz}, \mathrm{CDCl}_{3}\right) \delta: 7.98(\mathrm{~d}, J=$ $16.2 \mathrm{~Hz}, 1 \mathrm{H}), 7.52(\mathrm{~s}, 1 \mathrm{H}), 7.38(\mathrm{~d}, J=8.4 \mathrm{~Hz}, 1 \mathrm{H}), 6.94 \sim$ $6.86(\mathrm{~m}, 2 \mathrm{H}), 6.64(\mathrm{~d}, J=16.2 \mathrm{~Hz}, 1 \mathrm{H}), 4.31$ (q, $J=7.1$ $\mathrm{Hz}, 2 \mathrm{H}), 1.36(\mathrm{t}, J=7.1 \mathrm{~Hz}, 3 \mathrm{H}) ;{ }^{13} \mathrm{C}$ NMR $(101 \mathrm{MHz}$, $\left.\mathrm{CDCl}_{3}\right) \delta: 168.9,156.3,140.0,136.7,130.0,120.9,120.1$, $118.3,116.7,61.0,14.3$; HRMS (ESI) calcd for $\mathrm{C}_{11} \mathrm{H}_{11} \mathrm{Cl}-$ $\mathrm{NaO}_{3}[\mathrm{M}+\mathrm{Na}]^{+}$249.0294, found 249.0309 .

(E)-3-(5-(叔丁基)-2-毒基苯基)丙烯酸乙酯(3e): 28.3 $\mathrm{mg}$, 白色固体, 产率 57\%. m.p. $130 \sim 131{ }^{\circ} \mathrm{C} ;{ }^{1} \mathrm{H}$ NMR $\left(400 \mathrm{MHz}, \mathrm{CDCl}_{3}\right) \delta: 8.01(\mathrm{~d}, J=16.1 \mathrm{~Hz}, 1 \mathrm{H}), 7.48(\mathrm{~d}$, $J=2.4 \mathrm{~Hz}, 1 \mathrm{H}), 7.37 \sim 7.18(\mathrm{~m}, 1 \mathrm{H}), 6.80(\mathrm{~d}, J=8.5 \mathrm{~Hz}$, $1 \mathrm{H}), 6.65$ (d, $J=16.1 \mathrm{~Hz}, 1 \mathrm{H}), 6.13$ (s, 1H), 4.31 (q, $J=$ $7.1 \mathrm{~Hz}, 2 \mathrm{H}), 1.38$ (t, $J=7.1 \mathrm{~Hz}, 3 \mathrm{H}), 1.32(\mathrm{~s}, 9 \mathrm{H}) ;{ }^{13} \mathrm{C}$ NMR $\left(101 \mathrm{MHz}, \mathrm{CDCl}_{3}\right) \delta: 168.4,153.2,143.3,141.1$, 128.6, 125.9, 120.8, 118.1, 116.0, 60.6, 34.0, 31.3, 14.3; HRMS (ESI) calcd for $\mathrm{C}_{15} \mathrm{H}_{20} \mathrm{NaO}_{3}[\mathrm{M}+\mathrm{Na}]^{+} 271.1310$, found 271.1320 .

(E)-3-(5-丁基-2-羟基苯基)丙烯酸乙酯(3f): $29.3 \mathrm{mg}$, 白色固体, 产率 59\%. m.p. $124 \sim 127{ }^{\circ} \mathrm{C} ;{ }^{1} \mathrm{H}$ NMR (400 $\left.\mathrm{MHz}, \mathrm{CDCl}_{3}\right) \delta: 7.99(\mathrm{~d}, J=16.1 \mathrm{~Hz}, 1 \mathrm{H}), 7.26(\mathrm{~d}, J=3.1$ $\mathrm{Hz}, 1 \mathrm{H}), 7.06 \sim 7.01(\mathrm{~m}, 1 \mathrm{H}), 6.75(\mathrm{~d}, J=8.2 \mathrm{~Hz}, 1 \mathrm{H})$, $6.60(\mathrm{~d}, J=16.1 \mathrm{~Hz}, 1 \mathrm{H}), 6.25(\mathrm{~s}, 1 \mathrm{H}), 4.27(\mathrm{q}, J=7.1 \mathrm{~Hz}$, $2 \mathrm{H}), 2.55 \sim 2.48(\mathrm{~m}, 2 \mathrm{H}), 1.59 \sim 1.50(\mathrm{~m}, 2 \mathrm{H}), 1.36 \sim 1.29$ $(\mathrm{m}, 5 \mathrm{H}), 0.91(\mathrm{t}, J=7.3 \mathrm{~Hz}, 3 \mathrm{H}) ;{ }^{13} \mathrm{C}$ NMR $(101 \mathrm{MHz}$, $\left.\mathrm{CDCl}_{3}\right) \delta: 168.2,153.2,140.5,135.0,131.4,128.7,121.3$, 118.2, 116.2, 60.5, 34.6, 33.7, 22.2, 14.3, 13.9; HRMS (ESI) calcd for $\mathrm{C}_{15} \mathrm{H}_{20} \mathrm{NaO}_{3}[\mathrm{M}+\mathrm{Na}]^{+}$271.1310, found 271.1308.

(E)-3-(5-氟-2-羊基苯基)丙烯酸乙酯(3g): $22.3 \mathrm{mg}$, 白色固体, 产率 $53 \%$. m.p. $87 \sim 88{ }^{\circ} \mathrm{C}$ (文献值 ${ }^{[38]}$ : 88 $\left.90{ }^{\circ} \mathrm{C}\right) ;{ }^{1} \mathrm{H}$ NMR $\left(400 \mathrm{MHz}, \mathrm{CDCl}_{3}\right) \delta: 7.99 \sim 7.88(\mathrm{~m}$, $1 \mathrm{H}), 7.18 \sim 7.12(\mathrm{~m}, 1 \mathrm{H}), 6.99 \sim 6.90(\mathrm{~m}, 1 \mathrm{H}), 6.83 \sim 6.71$ $(\mathrm{m}, 1 \mathrm{H}), 6.54(\mathrm{~d}, J=16.1 \mathrm{~Hz}, 1 \mathrm{H}), 6.36(\mathrm{~s}, 1 \mathrm{H}), 4.28(\mathrm{q}$, $J=7.1 \mathrm{~Hz}, 2 \mathrm{H}), 1.35(\mathrm{t}, J=7.1 \mathrm{~Hz}, 3 \mathrm{H}) ;{ }^{13} \mathrm{C}$ NMR $(101$ $\left.\mathrm{MHz}, \mathrm{CDCl}_{3}\right) \delta: 167.8,156.8(\mathrm{~d}, J=238.8 \mathrm{~Hz}), 151.2(\mathrm{~d}$, $J=1.7 \mathrm{~Hz}), 139.1(\mathrm{~d}, J=2.1 \mathrm{~Hz}), 122.6(\mathrm{~d}, J=7.6 \mathrm{~Hz})$, $119.5,117.9$ (d, $J=23.4 \mathrm{~Hz}), 117.3$ (d, $J=8.0 \mathrm{~Hz}), 114.3$ (d, $J=23.5 \mathrm{~Hz}), 60.8,14.2 ;{ }^{19} \mathrm{~F}$ NMR $\left(376 \mathrm{MHz}, \mathrm{CDCl}_{3}\right)$ $\delta$ : -123.57 ; HRMS (ESI) calcd for $\mathrm{C}_{11} \mathrm{H}_{11} \mathrm{FNaO}_{3}[\mathrm{M}+$ $\mathrm{Na}]^{+}$233.0590, found 233.0580 .

(E)-3-(5-氯-2-羟基苯基)丙烯酸乙酯(3h): $25.8 \mathrm{mg}$, 白色固体, 产率 57\%. m.p. $101 \sim 103{ }^{\circ} \mathrm{C}$ (文献值 ${ }^{[38]}$ : $\left.102 \sim 104{ }^{\circ} \mathrm{C}\right) ;{ }^{1} \mathrm{H}$ NMR $\left(400 \mathrm{MHz}, \mathrm{CDCl}_{3}\right) \delta: 7.97(\mathrm{~d}$, $J=16.2 \mathrm{~Hz}, 1 \mathrm{H}), 7.45 \sim 7.41(\mathrm{~m}, 1 \mathrm{H}), 7.35(\mathrm{~s}, 1 \mathrm{H}), 7.20 \sim$ $7.15(\mathrm{~m}, 1 \mathrm{H}), 6.82(\mathrm{~d}, J=8.6 \mathrm{~Hz}, 1 \mathrm{H}), 6.62(\mathrm{~d}, J=16.2$ $\mathrm{Hz}, 1 \mathrm{H}), 4.30$ (q, $J=7.1 \mathrm{~Hz}, 2 \mathrm{H}), 1.35$ (t, $J=7.1 \mathrm{~Hz}, 3 \mathrm{H}$ ); ${ }^{13} \mathrm{C} \mathrm{NMR}\left(101 \mathrm{MHz}, \mathrm{CDCl}_{3}\right) \delta: 168.4,154.1,139.4,130.9$, 128.3, 125.4, 123.0, 119.2, 117.7, 60.9, 14.2; HRMS (ESI) calcd for $\mathrm{C}_{11} \mathrm{H}_{11} \mathrm{ClNaO}_{3}[\mathrm{M}+\mathrm{Na}]^{+}$249.0294, found 249.0306 .

(E)-3-(5-澳-2-羟基苯基)丙烯酸乙酯 (3i): $35.7 \mathrm{mg}$, 白色固体, 产率 $66 \%$. m.p. $108 \sim 109{ }^{\circ} \mathrm{C}$ (文献值 ${ }^{[38]}$ : $\left.108 \sim 110{ }^{\circ} \mathrm{C}\right) ;{ }^{1} \mathrm{H}$ NMR $\left(400 \mathrm{MHz}, \mathrm{CDCl}_{3}\right) \delta: 7.97(\mathrm{~d}, J=$ $16.2 \mathrm{~Hz}, 1 \mathrm{H}), 7.57(\mathrm{~d}, J=2.4 \mathrm{~Hz}, 2 \mathrm{H}), 7.33 \sim 7.28(\mathrm{~m}$, $1 \mathrm{H}), 6.78(\mathrm{~d}, J=8.6 \mathrm{~Hz}, 1 \mathrm{H}), 6.63(\mathrm{~d}, J=16.2 \mathrm{~Hz}, 1 \mathrm{H})$, 4.30 (q, $J=7.1 \mathrm{~Hz}, 2 \mathrm{H}), 1.35$ (t, $J=7.1 \mathrm{~Hz}, 3 \mathrm{H}) ;{ }^{13} \mathrm{C}$ NMR $\left(101 \mathrm{MHz}\right.$, DMSO- $\left.d_{6}\right) \delta: 164.4,153.9,136.3,131.8,128.8$, 121.0, 116.7, 116.2, 108.6, 57.9, 12.1; HRMS (ESI) calcd for $\mathrm{C}_{11} \mathrm{H}_{11} \mathrm{BrNaO}_{3}[\mathrm{M}+\mathrm{Na}]^{+}$292.9789, found 292.9810.

(E)-3-(2-羟基-5-(三氟甲基)苯基)丙烯酸乙酯 $(\mathbf{3} \mathbf{j})$ : $26.6 \mathrm{mg}$, 白色固体, 产率 51\%. m.p. $48 \sim 49{ }^{\circ} \mathrm{C} ;{ }^{1} \mathrm{H}$ NMR $\left(400 \mathrm{MHz}, \mathrm{CDCl}_{3}\right) \delta: 8.01(\mathrm{~d}, J=16.2 \mathrm{~Hz}, 1 \mathrm{H})$, $7.72 \sim 7.65(\mathrm{~m}, 1 \mathrm{H}), 7.53 \sim 7.41(\mathrm{~m}, 1 \mathrm{H}), 6.96(\mathrm{~d}, J=8.5$ $\mathrm{Hz}, 1 \mathrm{H}), 6.70(\mathrm{~d}, J=16.2 \mathrm{~Hz}, 1 \mathrm{H}), 4.31(\mathrm{q}, J=7.1 \mathrm{~Hz}$, $2 \mathrm{H}), 1.36(\mathrm{t}, J=7.1 \mathrm{~Hz}, 3 \mathrm{H}) ;{ }^{13} \mathrm{C} \mathrm{NMR}\left(101 \mathrm{MHz}, \mathrm{CDCl}_{3}\right)$ $\delta: 168.3,157.9,139.4,128.1$ (q, $J=3.4 \mathrm{~Hz}), 126.4$ (q, $J=$ $3.7 \mathrm{~Hz}), 123.0$ (dd, $J=31.1,5.4 \mathrm{~Hz}), 121.9,119.9,116.6$, 61.0, 14.2; ${ }^{19} \mathrm{~F}$ NMR (376 MHz, $\mathrm{CDCl}_{3}$ ) $\delta:-61.76$; HRMS (ESI) calcd for $\mathrm{C}_{12} \mathrm{H}_{11} \mathrm{~F}_{3} \mathrm{NaO}_{3} \quad[\mathrm{M}+\mathrm{Na}]$ 283.0558, found 283.0566.

(E)-3-(4-差基-(1,1'-联苯)-3-基)丙烯酸乙酯 (3k): $20.4 \mathrm{mg}$, 白色固体, 产率 38\%. m.p. $105 \sim 106{ }^{\circ} \mathrm{C} ;{ }^{1} \mathrm{H}$ NMR (400 MHz, $\left.\mathrm{CDCl}_{3}\right) \delta: 8.07(\mathrm{~d}, J=16.2 \mathrm{~Hz}, 1 \mathrm{H}), 7.70$ $(\mathrm{d}, J=2.3 \mathrm{~Hz}, 1 \mathrm{H}), 7.57 \sim 7.51(\mathrm{~m}, 2 \mathrm{H}), 7.49 \sim 7.39(\mathrm{~m}$, $3 \mathrm{H}), 7.36 \sim 7.30(\mathrm{~m}, 1 \mathrm{H}), 6.93(\mathrm{~d}, J=8.4 \mathrm{~Hz}, 1 \mathrm{H}), 6.70(\mathrm{~d}$, $J=16.1 \mathrm{~Hz}, 1 \mathrm{H}), 6.51(\mathrm{~s}, 1 \mathrm{H}), 4.30(\mathrm{q}, J=7.1 \mathrm{~Hz}, 2 \mathrm{H})$, $1.36(\mathrm{t}, J=7.1 \mathrm{~Hz}, 3 \mathrm{H}) ;{ }^{13} \mathrm{C} \mathrm{NMR}\left(101 \mathrm{MHz}, \mathrm{CDCl}_{3}\right) \delta$ : $168.2,154.9,140.4,140.2,133.9,130.0,128.7,127.7$, 126.9, 126.6, 121.9, 118.8, 116.8, 60.6, 14.3; HRMS (ESI) calcd for $\mathrm{C}_{17} \mathrm{H}_{17} \mathrm{O}_{3}[\mathrm{M}+\mathrm{H}]^{+}$269.1178, found 269.1183 .

(E)-3-(2-羟基-3-甲基苯基)丙烯酸乙酯(3I): $19.0 \mathrm{mg}$, 白色固体, 产率 $46 \%$. m.p. $66 \sim 68{ }^{\circ} \mathrm{C} ;{ }^{1} \mathrm{H}$ NMR $(400$ 
$\left.\mathrm{MHz}, \mathrm{CDCl}_{3}\right) \delta: 8.06(\mathrm{~d}, J=16.1 \mathrm{~Hz}, 1 \mathrm{H}), 7.35(\mathrm{~d}, J=7.8$ $\mathrm{Hz}, 1 \mathrm{H}), 7.17 \sim 7.13(\mathrm{~m}, 1 \mathrm{H}), 6.89 \sim 6.82(\mathrm{~m}, 1 \mathrm{H}), 6.52(\mathrm{~d}$, $J=16.1 \mathrm{~Hz}, 1 \mathrm{H}), 5.61(\mathrm{~s}, 1 \mathrm{H}), 4.28$ (q, $J=7.1 \mathrm{~Hz}, 2 \mathrm{H})$, $2.28(\mathrm{~s}, 3 \mathrm{H}), 1.34$ (t, $J=7.1 \mathrm{~Hz}, 3 \mathrm{H}) ;{ }^{13} \mathrm{C} \mathrm{NMR}(101 \mathrm{MHz}$, $\left.\mathrm{CDCl}_{3}\right) \delta: 168.0,153.5,140.4,132.7,126.3,124.1,121.6$, $120.5,118.3,60.5,15.9,14.3$; HRMS (ESI) calcd for $\mathrm{C}_{12} \mathrm{H}_{14} \mathrm{NaO}_{3}[\mathrm{M}+\mathrm{Na}]^{+}$229.0841, found 229.0833.

(E)-3-(2-羟基苯基)丙烯酸叔丁酯(4a): $27.8 \mathrm{mg}$, 白 色固体，产率 63\%. m.p. 98 $100{ }^{\circ} \mathrm{C} ;{ }^{1} \mathrm{H}$ NMR (400 $\left.\mathrm{MHz}, \mathrm{CDCl}_{3}\right) \delta: 8.03 \sim 7.95(\mathrm{~m}, 1 \mathrm{H}), 7.48 \sim 7.42(\mathrm{~m}, 1 \mathrm{H})$, $7.25 \sim 7.19(\mathrm{~m}, 2 \mathrm{H}), 6.91 \sim 6.84(\mathrm{~m}, 2 \mathrm{H}), 6.60 \sim 6.54(\mathrm{~m}$, $1 \mathrm{H}), 1.56(\mathrm{~s}, 9 \mathrm{H}) ;{ }^{13} \mathrm{C} \mathrm{NMR}\left(101 \mathrm{MHz}, \mathrm{CDCl}_{3}\right) \delta: 168.0$, $155.4,139.7,131.1,128.9,121.9,120.5,120.0,116.3$, 80.8, 28.2; HRMS (ESI) calcd for $\mathrm{C}_{13} \mathrm{H}_{16} \mathrm{NaO}_{3}[\mathrm{M}+\mathrm{Na}]^{+}$ 243.0997, found 243.1001.

(E)-3-(2-羟基苯基)丙烯酸苄酯(4b): $38.1 \mathrm{mg}$, 白色 固体, 产率 75\%. m.p. 86 88 ${ }^{\circ} \mathrm{C}$ (文献值 ${ }^{[41]}$ : $86 \sim 88{ }^{\circ} \mathrm{C}$ ); ${ }^{1} \mathrm{H}$ NMR (400 MHz, $\left.\mathrm{CDCl}_{3}\right) \delta: 8.01(\mathrm{~d}, J=16.2 \mathrm{~Hz}, 1 \mathrm{H})$, $7.39 \sim 7.25(\mathrm{~m}, 6 \mathrm{H}), 7.17 \sim 7.10(\mathrm{~m}, 1 \mathrm{H}), 6.81(\mathrm{~d}, J=8.0$ $\mathrm{Hz}, 2 \mathrm{H}), 6.73(\mathrm{~d}, J=8.1 \mathrm{~Hz}, 1 \mathrm{H}), 6.62$ (d, $J=16.2 \mathrm{~Hz}$, 1H), $5.20(\mathrm{~s}, 2 \mathrm{H}) ;{ }^{13} \mathrm{C} \mathrm{NMR}\left(101 \mathrm{MHz}, \mathrm{CDCl}_{3}\right) \delta: 168.3$, $155.4,141.3,135.9,131.5,129.3,128.5,128.2,121.5$, 120.6, 117.9, 116.4, 66.5; HRMS (ESI) calcd for $\mathrm{C}_{16} \mathrm{H}_{14^{-}}$ $\mathrm{NaO}_{3}[\mathrm{M}+\mathrm{Na}]^{+}$277.084, found 277.0850.

(E)-3-(2-羟基苯基)丙烯酸环己酯(4c): $46.3 \mathrm{mg}$, 淡 黄色液体, 产率 94\%. ${ }^{1} \mathrm{H} \mathrm{NMR}\left(400 \mathrm{MHz}, \mathrm{CDCl}_{3}\right) \delta: 8.05$ $(\mathrm{d}, J=16.2 \mathrm{~Hz}, 1 \mathrm{H}), 7.48 \sim 7.43(\mathrm{~m}, 1 \mathrm{H}), 7.42 \sim 7.27(\mathrm{~m}$, $1 \mathrm{H}), 7.25 \sim 7.20(\mathrm{~m}, 1 \mathrm{H}), 6.92 \sim 6.85(\mathrm{~m}, 2 \mathrm{H}), 6.65(\mathrm{~d}, J=$ $16.2 \mathrm{~Hz}, 1 \mathrm{H}), 4.96 \sim 4.89(\mathrm{~m}, 1 \mathrm{H}), 1.98 \sim 1.90(\mathrm{~m}, 2 \mathrm{H})$, $1.82 \sim 1.73(\mathrm{~m}, 2 \mathrm{H}), 1.62 \sim 1.44(\mathrm{~m}, 4 \mathrm{H}), 1.43 \sim 1.35(\mathrm{~m}$, $2 \mathrm{H}) ;{ }^{13} \mathrm{C}$ NMR (101 MHz, $\left.\mathrm{CDCl}_{3}\right) \delta: 168.1,155.6,140.6$, 131.3, 129.1, 121.7, 120.4, 118.7, 116.4, 73.0, 31.6, 25.3, 23.7; HRMS (ESI) calcd for $\mathrm{C}_{15} \mathrm{H}_{18} \mathrm{NaO}_{3}[\mathrm{M}+\mathrm{Na}]^{+}$ 269.1154 , found 269.1168 .

(E)-3-(2-差基-3-甲基苯基)丙烯酸烯丙酯(4d): 26.2 $\mathrm{mg}$, 淡黄色液体, 产率 $64 \% .{ }^{1} \mathrm{H} \mathrm{NMR}\left(400 \mathrm{MHz}, \mathrm{CDCl}_{3}\right.$ ) $\delta: 8.05(\mathrm{~d}, J=16.2 \mathrm{~Hz}, 1 \mathrm{H}), 7.49 \sim 7.46(\mathrm{~m}, 1 \mathrm{H}), 7.25 \sim$ $7.21(\mathrm{~m}, 1 \mathrm{H}), 6.96 \sim 6.90(\mathrm{~m}, 1 \mathrm{H}), 6.84(\mathrm{~d}, J=8.1 \mathrm{~Hz}$, $1 \mathrm{H}), 6.65(\mathrm{~d}, J=16.2 \mathrm{~Hz}, 1 \mathrm{H}), 6.42(\mathrm{~s}, 1 \mathrm{H}), 6.06 \sim 5.94$ $(\mathrm{m}, 1 \mathrm{H}), 5.43 \sim 5.35(\mathrm{~m}, 1 \mathrm{H}), 5.31 \sim 5.25(\mathrm{~m}, 1 \mathrm{H}), 4.77 \sim$ $4.71(\mathrm{~m}, 2 \mathrm{H}) ;{ }^{13} \mathrm{C} \mathrm{NMR}\left(101 \mathrm{MHz}, \mathrm{CDCl}_{3}\right) \delta: 167.7$, $155.2,140.7,132.2,131.4,129.2,121.6,120.8,118.2$, 118.2, 116.3, 65.2; HRMS (ESI) calcd for $\mathrm{C}_{15} \mathrm{H}_{12} \mathrm{NaO}_{3}$ $[\mathrm{M}+\mathrm{Na}]^{+}$227.0684, found 227.0687.

(E)-3-(2-羟基苯基)丙烯酸(全氟苯基)甲基酯(4e):
$46.1 \mathrm{mg}$, 白色固体, 产率 67\%. m.p. 132 134 ${ }^{\circ} \mathrm{C} ;{ }^{1} \mathrm{H}$ NMR (400 MHz, DMSO- $\left.d_{6}\right) \delta: 10.29$ (s, 1H), 7.88 (d, $J=$ $16.2 \mathrm{~Hz}, 1 \mathrm{H}), 7.62 \sim 7.53(\mathrm{~m}, 1 \mathrm{H}), 7.27 \sim 7.20(\mathrm{~m}, 1 \mathrm{H})$, $6.93 \sim 6.87(\mathrm{~m}, 1 \mathrm{H}), 6.84 \sim 6.77(\mathrm{~m}, 1 \mathrm{H}), 6.60(\mathrm{~d}, J=16.1$ $\mathrm{Hz}, 1 \mathrm{H}), 5.30$ (s, 2H); ${ }^{13} \mathrm{C}$ NMR (101 MHz, DMSO- $\left.d_{6}\right) \delta$ : 166.1, 157.0, 145.2 (dddd, $J=248.4,15.2,8.0,3.7 \mathrm{~Hz}$ ), 141.4, 141.0 (ddd, $J=12.9,9.1,5.1 \mathrm{~Hz}), 137.0(J=247.1$, 15.6, 10.6, 8.5, 5.0 4.9 Hz), 132.1, 129.1, 120.5, 119.4, 116.0 (d, $J=34.3 \mathrm{~Hz}), 110.1$ (td, $J=18.0,3.3 \mathrm{~Hz}), 53.0$; ${ }^{19} \mathrm{~F}$ NMR $\left(376 \mathrm{MHz}, \mathrm{DMSO}-d_{6}\right) \delta:-142.40$ (dd, $J=23.3$, $7.8 \mathrm{~Hz}),-153.59$ (t, $J=22.0 \mathrm{~Hz}),-162.47$ (dt, $J=23.0$, $7.9 \mathrm{~Hz}$ ); HRMS (ESI) calcd for $\mathrm{C}_{16} \mathrm{H}_{9} \mathrm{~F}_{5} \mathrm{NaO}_{3}[\mathrm{M}+\mathrm{Na}]^{+}$ 367.0370 , found 367.0360 .

(E)-3-(2-羟基苯基)- $N, N$-二甲基丙烯酰胺(4f)：27.2 $\mathrm{mg}$, 白色固体, 产率 71\%. m.p. 204 206 ${ }^{\circ} \mathrm{C}$ (文献值 ${ }^{[38]}$ : 205 207 ${ }^{\circ} \mathrm{C}$ ); ${ }^{1} \mathrm{H}$ NMR (400 MHz, DMSO-d $\left.d_{6}\right) \delta: 9.99$ (s, $1 \mathrm{H}), 7.73(\mathrm{~d}, J=15.6 \mathrm{~Hz}, 1 \mathrm{H}), 7.19 \sim 7.09$ (m, 1H), 6.88 $(\mathrm{d}, J=7.5 \mathrm{~Hz}, 2 \mathrm{H}), 6.84 \sim 6.78(\mathrm{~m}, 2 \mathrm{H}), 3.13(\mathrm{~s}, 3 \mathrm{H}), 2.92$ (s, 3H); ${ }^{13} \mathrm{C}$ NMR (101 MHz, DMSO) $\delta$ : 166.0, 156.1, 136.4, 130.6, 128.2, 121.9, 119.2, 117.3, 116.0, 36.8, 35.3; HRMS (ESI) calcd for $\mathrm{C}_{11} \mathrm{H}_{13} \mathrm{NNaO}_{2}[\mathrm{M}+\mathrm{Na}]^{+}$ 214.0844, found 214.0843.

(E)-2-(2-(苯磺酰基)乙烯基)苯酚(4g)：31.2 mg, 白 色固体, 产率 $60 \%$. m.p. $160 \sim 162{ }^{\circ} \mathrm{C}$ (文献值 ${ }^{[42]}: 159 \sim$ $\left.162{ }^{\circ} \mathrm{C}\right) ;{ }^{1} \mathrm{H}$ NMR $\left(400 \mathrm{MHz}, \mathrm{CDCl}_{3}\right) \delta: 8.02 \sim 7.97(\mathrm{~m}$, $1 \mathrm{H}), 7.92(\mathrm{~d}, J=15.5 \mathrm{~Hz}, 1 \mathrm{H}), 7.70 \sim 7.64(\mathrm{~m}, 1 \mathrm{H}), 7.62 \sim$ $7.56(\mathrm{~m}, 1 \mathrm{H}), 7.46 \sim 7.41(\mathrm{~m}, 1 \mathrm{H}), 7.31(\mathrm{~d}, J=8.2 \mathrm{~Hz}$, $1 \mathrm{H}), 7.01 \sim 6.96(\mathrm{~m}, 1 \mathrm{H}), 6.92(\mathrm{~d}, J=8.1 \mathrm{~Hz}, 1 \mathrm{H}), 6.71(\mathrm{~s}$, $1 \mathrm{H}) ;{ }^{13} \mathrm{C}$ NMR (101 MHz, DMSO-d $\left.d_{6}\right) \delta: 157.2,141.1$, 137.6, 133.4, 132.7, 129.8, 129.6, 127.1, 126.8, 119.5, 118.9, 116.3; HRMS (ESI) calcd for $\mathrm{C}_{14} \mathrm{H}_{12} \mathrm{NaO}_{3} \mathrm{~S}[\mathrm{M}+$ $\mathrm{Na}]^{+}$283.0405, found 283.0419 .

(E)-2-苯乙烯基苯酚(4h)：18.9 mg，白色固体，产率 48\%. m.p. 53 55 ${ }^{\circ} \mathrm{C}$; ${ }^{1} \mathrm{H}$ NMR (400 MHz, $\left.\mathrm{CDCl}_{3}\right) \delta$ : $7.69(\mathrm{~d}, J=7.7 \mathrm{~Hz}, 3 \mathrm{H}), 7.55 \sim 7.50(\mathrm{~m}, 2 \mathrm{H}), 7.44 \sim 7.39$ $(\mathrm{m}, 2 \mathrm{H}), 7.34 \sim 7.28(\mathrm{~m}, 2 \mathrm{H}), 7.11(\mathrm{t}, J=7.6 \mathrm{~Hz}, 1 \mathrm{H}), 6.97$ $(\mathrm{d}, J=8.1 \mathrm{~Hz}, 1 \mathrm{H}), 5.26(\mathrm{~s}, 1 \mathrm{H}) ;{ }^{13} \mathrm{C} \mathrm{NMR}(101 \mathrm{MHz}$, $\left.\mathrm{CDCl}_{3}\right) \delta: 153.0,137.5,130.1,128.6,127.5,127.1,126.5$, 124.6, 123.0, 121.1, 115.9; HRMS (ESI) calcd for $\mathrm{C}_{14} \mathrm{H}_{12} \mathrm{NaO}[\mathrm{M}+\mathrm{Na}]^{+}$219.0786, found 219.0779.

(E)-2,2,3,3,4,4,5,5,6,6,7,7,8,8,8-十五氟辛基 3-(2-羊圣 基苯基)丙烯酸酯(4i): $43.7 \mathrm{mg}$, 白色固体, 产率 $40 \%$. m.p. $117 \sim 118{ }^{\circ} \mathrm{C} ;{ }^{1} \mathrm{H}$ NMR (400 MHz, DMSO- $\left.d_{6}\right) \delta$ : 10.37 (s, 1H), 7.97 (d, $J=16.1 \mathrm{~Hz}, 1 \mathrm{H}), 7.60$ (d, $J=7.6$ $\mathrm{Hz}, 1 \mathrm{H}), 7.32 \sim 7.22(\mathrm{~m}, 1 \mathrm{H}), 6.92(\mathrm{~d}, J=8.2 \mathrm{~Hz}, 1 \mathrm{H})$, 
$6.85 \sim 6.78(\mathrm{~m}, 1 \mathrm{H}), 6.66(\mathrm{~d}, J=16.1 \mathrm{~Hz}, 1 \mathrm{H}), 4.91(\mathrm{t}, J=$ $14.4 \mathrm{~Hz}, 2 \mathrm{H}) ;{ }^{13} \mathrm{C}$ NMR (101 MHz, DMSO- $\left.d_{6}\right) \delta: 165.2$, 157.3, 142.6, 132.3, $130.9(J=316.3,11.1 \mathrm{~Hz}), 129.1$, 120.3, 119.3, $117.1(J=473.7,9.6 \mathrm{~Hz}), 116.3,114.6,112.0$ $(J=77.9,6.1 \mathrm{~Hz}), 109.5(J=278.3,3.4 \mathrm{~Hz}), 106.5(J=$ $184.4,179.2 \mathrm{~Hz}), 58.6(\mathrm{ddd}, J=17.7,11.1,5.7 \mathrm{~Hz}) ;{ }^{19} \mathrm{~F}$ NMR $\left(376 \mathrm{MHz}, \mathrm{CDCl}_{3}\right) \delta:-80.73(\mathrm{t}, J=9.9 \mathrm{~Hz})$, $-119.34(\mathrm{~d}, J=4.2 \mathrm{~Hz}),-121.94,-122.69,-123.24$, - 126.08; HRMS (ESI) calcd for $\mathrm{C}_{17} \mathrm{H}_{9} \mathrm{~F}_{15} \mathrm{NaO}_{3}[\mathrm{M}+\mathrm{Na}]^{+}$ 569.0210 , found 569.0228 .

(E)-3-(2-羟基苯基)丙烯酸十二烷基酯 $(4 \mathbf{j})$ : $38.6 \mathrm{mg}$, 白色固体，产率 58\%. m.p. 48 49 ${ }^{\circ} \mathrm{C} ;{ }^{1} \mathrm{H}$ NMR (400 $\left.\mathrm{MHz} \mathrm{CDCl}_{3}\right) \delta: 8.05(\mathrm{~d}, J=16.2 \mathrm{~Hz}, 1 \mathrm{H}), 7.49 \sim 7.44(\mathrm{~m}$, $1 \mathrm{H}), 7.26 \sim 7.20(\mathrm{~m}, 1 \mathrm{H}), 7.11 \sim 7.05(\mathrm{~m}, 1 \mathrm{H}), 6.93 \sim 6.84$ $(\mathrm{m}, 2 \mathrm{H}), 6.65(\mathrm{~d}, J=16.2 \mathrm{~Hz}, 1 \mathrm{H}), 4.23(\mathrm{t}, J=6.8 \mathrm{~Hz}, 2 \mathrm{H})$, $1.76 \sim 1.67(\mathrm{~m}, 2 \mathrm{H}), 1.33 \sim 1.24(\mathrm{~m}, 18 \mathrm{H}), 0.88(\mathrm{t}, J=6.8$ $\mathrm{Hz}, 3 \mathrm{H}) ;{ }^{13} \mathrm{C} \mathrm{NMR}\left(101 \mathrm{MHz}, \mathrm{CDCl}_{3}\right) \delta: 168.6,155.5$, 140.7, 131.3, 129.1, 121.7, 120.5, 118.2, 116.4, 64.9, 63.1, 32.6, 31.9, 29.7, 29.6, 29.3, 28.7, 25.9, 25.6, 22.6, 14.1; HRMS (ESI) calcd for $\mathrm{C}_{21} \mathrm{H}_{32} \mathrm{NaO}_{3}[\mathrm{M}+\mathrm{Na}]^{+} 355.2249$, found 355.2261 .

\section{4 分子间竞争的 KIE 实验}

向 $15 \mathrm{~mL}$ 的玻璃反应管中依次加入 $\left[\mathrm{Cp}^{*} \mathrm{RhCl}_{2}\right]_{2}(5$ mol\%, $6.2 \mathrm{mg}), \mathrm{AgOAc}$ (0.2 mmol, 1 equiv.), 丙酮肟苯醚 (0.1 mmol，0.5 equiv.), 氝代丙酮肜苯醚 $(0.1 \mathrm{mmol}, 0.5$ equiv.), 丙烯酸乙酯( $0.4 \mathrm{mmol}, 2$ equiv.)和 $1 \mathrm{~mL}$ 甲醇, 将 反应管密封, 在 $80{ }^{\circ} \mathrm{C}$ 下反应 $2 \mathrm{~h}$. 取出反应管, 旋干溶 剂, 粗产物用硅胶柱层析提纯, 淋洗剂为石油醚和乙酸 乙酯的混合溶剂 $[V$ (石油醚 $): V($ 乙酸乙酯 $)=8 ： 1]$. 通 过核磁分析得出 KIE 值为 2.6.

辅助材料(Supporting Information) 化合物 $3 \mathrm{a} \sim 31$ 和 $\mathbf{4 a} \sim \mathbf{4 j}$ 的 ${ }^{1} \mathrm{H}$ NMR 和 ${ }^{13} \mathrm{C}$ NMR 图谱. 这些材料可以免费 从本刊网站(http://sioc-journal.cn/)上下载.

\section{References}

[1] (a) Zhou, P. P.; Guan, M. Y.; Zhang, J. Y.; Xu, F.; Zhao, Y. S. Chin. J. Org. Chem. 2017, 37, 2028 (in Chinese).

(周盼盼，管明玉，张静宇，徐凡，赵应声，有机化学， 2017，37, 2028.)

(b) Zheng, Y. X.; Han, J.; Huang, Z. B.; Shi, D. Q.; Zhao, Y. S. Chin. J. Org. Chem. 2017, 37, 2066 (in Chinese).

(郑永祥, 韩健, 黄志斌, 史达清, 赵应声, 有机化学, 2017, 37, 2066.)

(c) Shang, X. J.; Liu, Z. Q. Chin. J. Org. Chem. 2015, 35, 522 (in Chinese).

(尚䈠洁，柳忠全，有机化学，2015, 35, 522.)

(d) Luo, F. H.; Long, Y.; Li, Z. K.; Zhou, X. G. Acta Chim. Sinica 2016, 74, 805 (in Chinese).

(罗飞华, 龙洋, 李正凯, 周向葛, 化学学报, 2016, 74, 805.)
[2] (a) Gou, Q.; Tan, X. P.; Zhang, M. Z.; Ran, M.; Yuan, T. R.; He, S. H.; Zhou, L. Z.; Cao, T. W.; Luo, F. H. Org. Lett. 2020, 22, 1966. (b) Dai, J.-L.; Shao, N.-Q.; Zhang, J.; Jia, R.-P.; Wang, D.-H. J. Am. Chem. Soc. 2017, 139, 12393.

(c) Fabry, D. C.; Ronge, M. A.; Zoller, J.; Rueping, M. Angew. Chem., Int. Ed. 2015, 54, 2801.

(d) Zhang, L.; Zhu, L.; Zhang, Y.; Yang, Y.; Wu, Y.; Ma, W.; Lan, Y.; You, J. ACS Catal. 2018, 8. 8324.

(e) Khaefa, S.; Rostamic, A.; Khakyzadehd, V.; Zolfigolb, M. A.; Taherpoura, A. A.; Yarieb, M. Mol. Catal. 2020, 484, 110772.

[3] Boele, M. D. K.; van Strijdonck, G. P. F.; de Vries, A. H. M.; Kamer, P. C. J.; de Veries J.-G.; van Leeuwen, P. W. N. M. J. Am. Chem. Soc. 2002, 124, 1586

[4] Beck, E. M.; Grimster, N. P.; Hatley, R.; Gaunt, M. J. J. Am. Chem Soc. 2006, 128, 2528.

[5] Wu, J. L.; Cui, X. L.; Chen, L. M.; Jiang, G. J.; Wu, Y. G. J. Am. Chem. Soc. 2009, 131, 13888.

[6] Wang, D. H.; Engle, K. M.; Shi, B.-F.; Yu, J.-Q. Science 2010, 327 , 315.

[7] Xu, W. T.; Wang, N.; Zhang, M. Y.; Shi, D. Q. Chin. J. Org. Chem. 2019, 39, 1735 (in Chinese) (徐文韬，王宁，张梦烨，史达清，有机化学，2019，39，1735.)

[8] Ackermann, L.; Pospech, J. Org. Lett. 2011, 13, 4153.

[9] Padala, K.; Jeganmohan, M. Org. Lett. 2011, 13, 6144.

[10] Hashimoto, Y.; Hirano, K.; Satoh, T.; Kakiuchi, F.; Miura, M. Org. Lett. 2012, 14, 2058.

[11] Manikandan, R.; Madasamy, P.; Jeganmohan, M. ACS Catal. 2016, 6,230 .

[12] Dana, S.; Mandal, A.; Sahoo, H.; Mallik, S.; Grandhi, G.-S.; Baidya, M. Org. Lett. 2018, 20, 716.

[13] Patureau, F. W.; Glorius, F. J. Am. Chem. Soc. 2010, 132, 9982.

[14] Yin, B.; Fu, M.-L.; Zhu, Q. Chin. J. Org. Chem. 2020, 40, 1461 (in Chinese).

(尹彪, 付曼琳, 朱就, 有机化学, 2020, 40, 1461.)

[15] Wang, C. M.; Chen, H.; Wang, Z. F.; Chen, J. A.; Huang, Y. Angew. Chem., Int. Ed. 2012, 51, 7242.

[16] Kona, C. N.; Nishii, Y.; Miura, M. Org. Lett. 2018, 20, 4898.

[17] Gan, F. F.; Chua, Y. S.; Scarmagnani, S.; Palaniappan, P.; Franks, M.; Poobalasingam, T.; Bradshaw, T. D.; Westwell, A. D.; Hagen, T. Res. Commun. 2009, 387, 741.

[18] Tyman, J. H. P. Synthetic and Natural Phenols, Elsevier, New York, 1996.

[19] Finkelstein, B. L.; Benner, E. A.; Hendrixson, M. C.; Kranis, K. T.; Rauh, J. J.; Sethuraman, M. R.; McCann, S. F. Bioorg. Med. Chem. 2002, 10,599

[20] Martín Castro, A. M. Chem. Rev. 2004, 104, 2939.

[21] (a) Reddy, M. C.; Jeganmohan, M. Eur. J. Org. Chem. 2013, 1150. (b) Li, B.; Ma, J.; Liang, Y.; Wang, N.; Xu, S.; Song, H.; Wang, B. Eur. J. Org. Chem. 2013, 1950

(c) Dai, H.-X.; Li, G.; Zhang, X.-G.; Stepan, A. F.; Yu, J.-Q. J. Am. Chem. Soc. 2013, 135, 7567.

[22] Huang, C. H.; Chattopadhyay, B.; Gevorgyan, V. J. Am. Chem. Soc. 2011, 133, 12406

[23] Gong, T.-J.; Xiao, B.; Liu, Z.-J.; Wan, J.; Xu, J.; Luo, D.-F.; Fu, Y.; Liu, L. Org. Lett. 2011, 13, 3235 .

[24] Shen, Y. Y.; Liu, G. X.; Zhou, Z.; Lu, X. Y. Org. Lett. 2013, 15, 3366.

[25] Bolotin, D. S.; Bokach, N. A.; Demakova, M. Y.; Kukushkin, V. Y.; Chem. Rev. 2017, 117, 13039.

[26] Singh, N.; Karpichev, Y.; Tiwari, A. K.; Kuca, K.; Ghosh, K. K.; J. Mol. Liq. 2015, 208, 307.

[27] Worek, F.; Thiermann, H.; Wille, T. Chem.-Biol. Interact. 2016, $259,93$.

[28] Guo, K.; Chen, X. L.; Guan, M. Y.; Zhao, Y. S. Org. Lett. 2015, 17, 1802 .

[29] Liu, L. L.; Wang, N.; Dai, C. Y.; Han, Y.; Yang, S.; Huang, Z. B.; Zhao, Y. S. Eur. J. Org. Chem. 2019, 7857.

[30] Wang, N.; Liu, L. L.; Xu, W. T.; Zhang, M. Y.; Huang, Z. B.; Shi, 
D. Q.; Zhao, Y. S. Org. Lett. 2019, 21, 365.

[31] Zhou, B.; Chen, Z. Q.; Yang, Y. X.; Ai, W.; Tang, H. Y.; Wu, Y. X.; Zhu, W. L.; Li, Y. C. Angew. Chem., Int. Ed. 2015, 54, 12121.

[32] Xie, F.; Qi, Z. S.; Yu, S. J.; Li, X. W. J. Am. Chem. Soc. 2014, 136, 4780 .

[33] Asaumi, T.; Matsuo, T.; Fukuyama, T.; Ie, Y.; Kakiuchi, F.; Chatani, N. J. Org. Chem. 2004, 69, 4433.

[34] Hyster, T. K.; Rovis, T. J. Am. Chem. Soc. 2010, 132, 10565.

[35] Liu, B.; Fan, Y.; Gao, Y.; Sun, C.; Xu, C.; Zhu, J. J. Am. Chem. Soc. 2013, 135, 468.

[36] Xu, L.; Zhu, Q.; Huang, G.; Cheng, B.; Xia, Y. J. Org. Chem. 2012,
77, 3017.

[37] Takeda, N.; Miyata, O.; Naito, T. Eur. J. Org. Chem. 2007, 1491.

[38] Rao, M. L. N.; Ramakrishna, B. S. J. Org. Chem. 2019, 84, 5677.

[39] Liu, B.; Jiang, H. Z.; Shi, B. F. J. Org. Chem. 2014, 79, 1521.

[40] Chen, J. M.; Liu, W.; Zhou, L. D.; Zhao, Y. L. Tetrahedron Lett. 2018, 59, 2526.

[41] Ribeiro Laia, F. M.; Pinho e Melo, T. M. V. D. Synthesis 2015, 47, 2781.

[42] Jiang, Q.; Xu, B.; Jia, J.; Zhao, A.; Zhao, Y. R.; Li, Y. Y.; He, N. N.; Guo, C. C. J. Org. Chem. 2014, 79, 7372.

(Zhao, C.) 